\title{
Quantitative Approach to Single-Nucleotide Polymorphism Analysis Using MALDI-TOF Mass Spectrometry
}

BioTechniques 29:620-629(September 2000)

\author{
Philip Ross, Laura Hall and \\ Lawrence A. Haff \\ Applied Biosytems, \\ Framingham, MA, USA
}

\section{INTRODUCTION}

Current large-scale genome sequencing efforts continue to uncover singlenucleotide polymorphisms (SNPs) with a frequency of approximately one SNP/ kb DNA $(9,18,24,27)$. As SNPs are used in applications such as gene localization, drug resistance testing, disease diagnostics or agricultural trait enhancement, a concomitant increase in the rate of routine SNP characterization will be necessary. Pooling of DNA samples offers a method to reduce the number of genotypes that must be obtained $(2,4,8,17,20,26)$. In principle, information derived from the analysis of amplified DNA pools that harbor sequence variants can be used to determine allele frequencies across selected groups or populations, validate a candidate SNP as common or rare or merely detect the presence of a particular variant in collections of DNA samples.

For analysis of pooled, amplified SNP loci, the challenge is to preserve a sufficiently high level of specificity, sensitivity and economy while maintaining acceptable throughput. An assortment of potentially viable highthroughput SNP genotyping technologies has emerged, each with its own advantages and disadvantages. These include oligonucleotide arrays, $5^{\prime} \mathrm{nu}-$ clease (TaqMan ${ }^{\circledR}$ ) assays, oligonucleotide ligation and various single-base primer extension formats. Quantitative usage of the array and $5^{\prime}$ nuclease approaches has been very succesful for the measurement of gene expression or viral contamination. However, only the oligonucleotide ligation $(3,10,16)$, single-base extension and more recently the $5^{\prime}$ nuclease assays (5) have been documented as quantifying a point mutation or SNP allele in a background of wild-type DNA $(12,22,23)$.

Analysis of primer extension assays by delayed extraction, matrix-assisted laser desorption ionization time-offlight mass spectrometry (MALDI-TOF MS) (15) offers a flexible, low-cost and highly specific approach to SNP analysis. Recently, a MALDI-TOF-based genotyping approach was introduced in which genotypes were determined by accurate molecular weight measurement of genotyping primers extended by a single dideoxynucleotide $(13,14$, 21 ). The assay uses a single unlabeled primer to resolve all possible SNP alleles in a low-volume, post-PCR that can be multiplexed to a level of 12 or more loci per reaction $(14,21)$. Although a basis for quantitative MALDI-TOF MS analysis of synthetic oligonucleotides has been documented (7), an investigation into the quantitative ability of mass spectrometry in the context of genetic analysis has not been presented. Ultimately, a MALDI-TOF platform that enables low-cost, high-throughput genotyping will be further enhanced by the ability to perform quantitative measurements in DNA pools.

In this paper, we explore the basic capabilities of MALDI-TOF MS as a tool for quantitative SNP analysis in DNA pools. In particular, we evaluate the quantitative ranges and detection 
limits using standard mixtures of PCR products from several well-characterized SNP loci. The samples are genotyped using two related primer extension protocols that produce final reaction products differing in mass by 300-400 Da between alleles to aid in peak area measurement. Analysis of mixtures of heterozygote DNA in varied excess amounts of homozygous wild-type DNA reveals a limit of detection (LOD) of $2 \%$ and a limit of quantitation (LOQ) of $5 \%-10 \%$ for the variant allele. These results support the applicability of MALDI-TOF for studies of pooled DNA using the approaches described here.

\section{MATERIALS AND METHODS}

\section{PCR Amplification}

PCR was carried out in $25-\mu \mathrm{L}$ volumes composed of 25 pmol each primer, $1 \times$ PCR buffer II (PE Biosystems, Foster City, CA, USA), $2.5 \mathrm{mM}$ $\mathrm{MgCl}_{2}, 200 \mu \mathrm{M}$ dNTPs, $50 \mathrm{ng}$ genomic DNA, $0.5 \mathrm{U}$ AmpliTaq ${ }^{\circledR}$ Gold (PE Biosystems). The reactions were performed using a PE Biosytems 9600 or 2400 DNA Thermal Cycler according to the following program: $95^{\circ} \mathrm{C}$ for 10 min, 30 cycles of $94^{\circ} \mathrm{C}$ for $1 \mathrm{~min}, 60^{\circ} \mathrm{C}$ for $30 \mathrm{~s}$ and $72^{\circ} \mathrm{C}$ for $60 \mathrm{~s}$, followed by a 7 -min incubation at $72^{\circ} \mathrm{C}$. Reactions were stored at $4^{\circ} \mathrm{C}$ before use. PCR products were quantitated by fluorescence using SYBR Green ${ }^{\circledR}$ dye (BMA, Rockland ME, USA) on a CytoFluor ${ }^{\circledR}$ fluorescence plate reader (PE Biosystems). PCR product pools and dilutions were prepared by diluting a known heterozygote PCR product into PCR product from homozygous individuals to obtain the desired concentrations.

\section{Primer Extension Assay}

The procedure used here was based on previously described primer extension assays $(13,14,21)$. PCR product mixtures $(10 \mu \mathrm{L})$ were treated with 10 $\mathrm{U}$ shrimp alkaline phosphatase and $1 \mathrm{U}$ exonuclease I (both from Amersham Pharmacia Biotech, Piscataway, NJ, USA) and incubated for $15 \mathrm{~min}$ at $37^{\circ} \mathrm{C}$, then for $15 \mathrm{~min}$ at $85^{\circ} \mathrm{C}$. Primer extension reaction mixture $(5 \mu \mathrm{L})$, comprising $1 \mathrm{U}$ AmpliTaq FS DNA polymerase, 10 pmol genotyping primer, $0.8 \times$ PCR II buffer, $4 \mathrm{mM}$ $\mathrm{MgCl}_{2}$ and nucleotides, was added to 5 $\mu \mathrm{L}$ treated PCR product. For genotyping pools of cytochrome P450 (CP450) and low-density lipoprotein receptor (LDLR) PCR products, the nucleotides were ddATP, ddGTP, dTTP and ddCTP at $200 \mathrm{mM}$ (final concentration), while for genotyping aldolase B (ALDOB) PCR products, the reactions contained ddCTP, ddGTP, ddTTP and FAM-labeled ddATP $\left(\mathrm{NEN}^{\circledR}\right.$ Life Science Products, Boston, MA, USA) at final concentrations of $5 \mu \mathrm{M}$ each. Primer extension reactions were then performed as follows: 25 cycles of $94^{\circ} \mathrm{C}$ for $1 \mathrm{~min}, 37^{\circ} \mathrm{C}$ for $30 \mathrm{~s}$ and $70^{\circ} \mathrm{C}$ for $60 \mathrm{~s}$ and then desalted.

\section{Mass Spectrometry}

Samples were desalted before analysis using a reverse-phase absorption elution procedure (13) in 96-well, lowvolume filter microplates. Aliquots of the desalted sample were combined with an equal volume of matrix solution $(50 \mathrm{mg} / \mathrm{mL}$ 3-hydroxy picolinic acid, $10 \mathrm{mg} / \mathrm{mL}$ picolinic acid and 50 $\mathrm{mM}$ ammonium citrate) and transferred in 300-400 nL quantities to 400- or 384-well MALDI plates (PE Biosystems) masked with a hydrophobic polymer coating. All mass spectrometry was performed on a Voyager $^{\mathrm{TM}} \mathrm{DE}$ Linear benchtop MALDI-TOF mass spectrometer (PE Biosystems) operating in positive-ion, delayed extraction mode. Typically, spectra were accumulated for 50 laser shots and stored. To measure peak areas, all spectra were treated by an identical series of processing steps using Data Explorer ${ }^{\mathrm{TM}}$ (PE Biosystems) software. Spectra were internally calibrated using an unextended primer as a single-point standard, and then a baseline correction function was applied, followed by data smoothing using a 21-point Gaussian filter function. A universal set of peak detection settings was created through the Data Explorer software and applied to all spectra. These settings define the minimum width, resolution, noise level and minimum area with which to discriminate peaks from background. With the appropriate peak detection 
settings, area contributions from salt adducts are rejected. Peak detection, peak area measurement and signal-tonoise $(\mathrm{S} / \mathrm{N})$ measurement were then performed using these parameters. Taken together, these parameters offer several degrees of noise and artifact rejection such that peak detection and peak areas can be measured reliably down to an $\mathrm{S} / \mathrm{N}$ of 3 .

\section{RESULTS}

To investigate the sensitivity and specificity of allele detection in pooled PCR products, SNPs from the CP450 2D6 gene (1), LDLR gene (25) and ALDOB gene (6) were selected as model systems. Accurate quantitation of an allele in the presence of an excess of another requires accurate determination of peak areas. Single-base primer extension by the addition of dideoxynucleotides produces genotype peaks differing by 9-40 Da. Two possible factors may contribute error during mass spectral peak area measurement. First, in routine analysis where minimal attention is paid to instrument optimization, baseline resolution between alleles differing by $16 \mathrm{Da}$ or less may not be observed. For data in which there is a low proportion of one allele, partial resolution of neighboring peaks with disparate areas may pose problems for reliable peak detection and correct area measurement. Second, area measurement of a low-intensity extension product within $40 \mathrm{Da}$ of another allele may be confounded by trace cation $\left(\mathrm{NH}_{4}^{+}, \mathrm{Na}^{+}\right.$, $\mathrm{K}^{+}$) adducts onto the lower mass allele.

For these reasons, two related strategies were selected by which a molecular weight separation of about 300-400 Da between allele products of a given locus could be achieved during the primer extension assay. In so doing, both the resolution and low-level adduct problems are circumvented. Both procedures use a modified form of a thermal-cycled single-base primer extension procedure described previously for testing point mutations on PCR products. The details of the modified assays are shown in Figure 1. The changes to the single-base extension assay implemented in the present work retain the basic desirable features of the

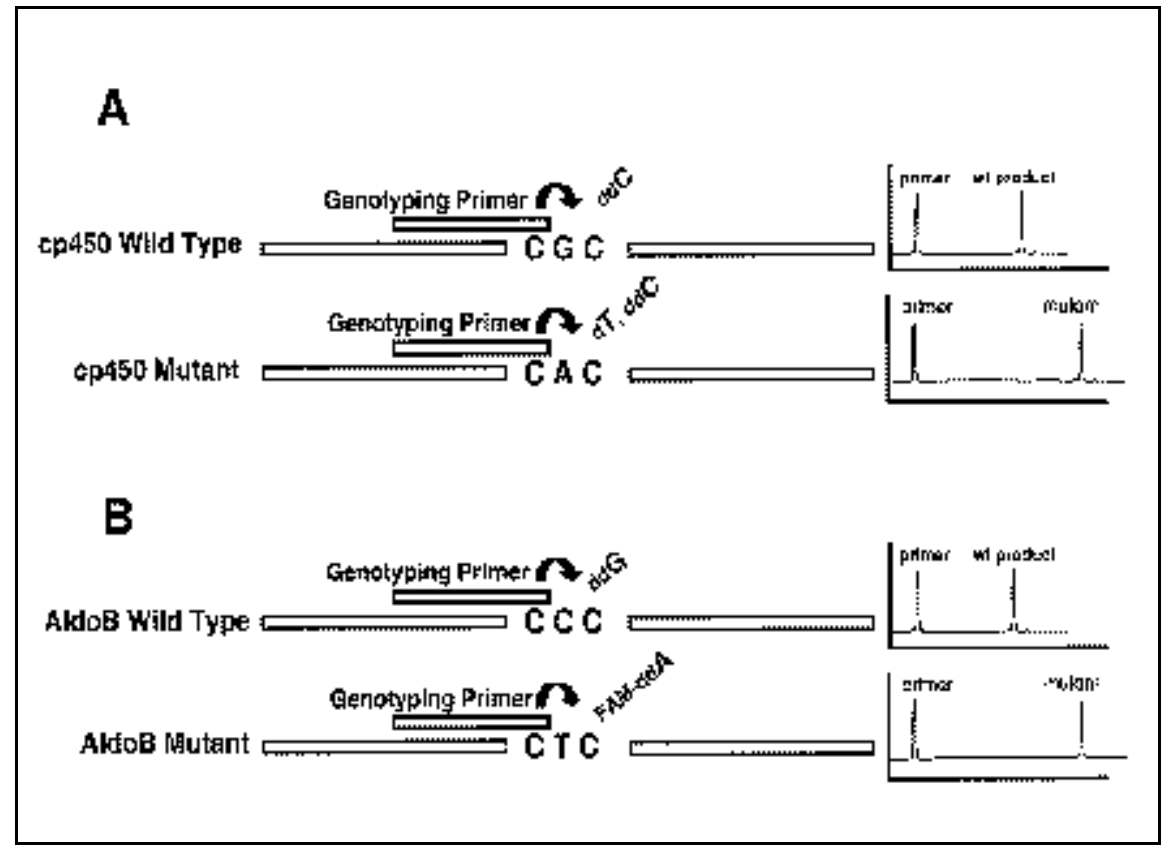

Figure 1. Schematic illustration of primer extension assays used to quantitate SNP alleles in DNA pools. (A) The CP450 wild-type and mutant alleles generate primers extended by ddC and dT $+\mathrm{ddC}$, respectively (mass difference $=304.2 \mathrm{Da}$ ), from a reaction mixture containing dT, ddC, ddA and ddG. $(\mathrm{B})$ When a reaction mixture containing ddG, ddC, ddT and FAM-ddA is used, wild-type and mutant alleles yield products extended by ddG and FAM-ddA, respectively (mass difference $=410 \mathrm{Da}$ ).
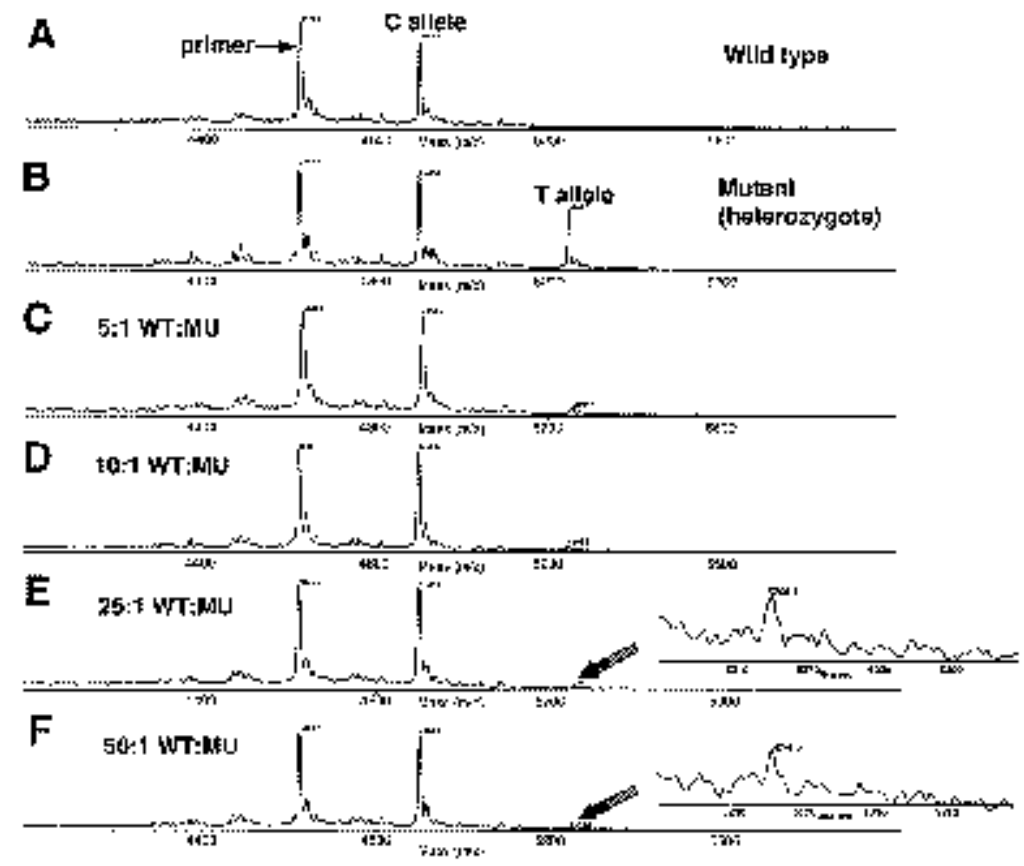

Figure 2. Mass spectra obtained at representative intervals from a quantitation study of CP450 2D6 SNP site in pooled PCR products. Oligonucleotide sequences used were: PCR F: 5'CCAAGGGGAACCCTGAGAG-3', PCR R: 5'-GTGGTCGAGGTGGTCACCATC-3', genotyping primer: 5'-AATGATGAGAACCTG-3'. Mass spectra were generated from homozygous wild-type (A), heterozygous mutant (B) and mixtures of PCR from wild-type individuals and heterozygous mutant individuals in wild-type:mutant ratios ranging from 5:1-50:1 (C-F). 
previously described assay $(13,14,21)$ (i) no off-line PCR product handling or purification is required, (ii) the assay is performed in a single tube, (iii) thermal cycling is used to linearly amplify product yield, (iv) a single primer can discriminate all possible genotypes and (v) one or both PCR product strands can be used as template.

In the first approach, a mixture of deoxy- and dideoxynucleotides is used to generate wild-type and mutant products that are terminated after extension by one (wild-type) or two (mutant) bases. Using a mixture of dT, ddA, ddG and $\mathrm{ddC}$ with the CP450 genotyping primer generates a wild-type allele that has extended by dT and a mutant allele corresponding to a $\mathrm{C} \rightarrow \mathrm{T}$ substitution that extends by ddC and dT. In the second approach, as tested with the ALDOB example (Figure 1B), an assay mixture containing ddC, ddG, ddT and FAM labeled ddA was used. In this way, the mutant A allele has a molecular weight difference of 410 Da from the $\mathrm{G}$ wild-type. The use of mixtures of deoxy- and dideoxynucleotides, as well as fluorescently labeled dideoxynucleotides in mass spectrometry-based primer extension assays, has been previously reported $(11,19)$.

Typical assays for CP450 2D6 PCR products from homozygote and heterozygote samples are shown in Figure 2, $\mathrm{A}$ and $\mathrm{B}$, respectively. To perform quantitation, the average peak area ratio between $\mathrm{C}$ and $\mathrm{T}$ alleles in a known heterozygote can establish a response factor to be applied in subsequent measurements. By normalizing slight differences in extension yield in this way, quantitation does not rely on equal peak areas for a 50/50 heterozygote. A quantitation study was performed by combining heterozygous CP450 PCR product with increasing amounts of homozygous PCR products (Figure 2, C-F). Accordingly, a steady decrease in the amount of the corresponding $\mathrm{T}$ allele was observed in the mass spectra. Relative peak areas were calculated for five replicate spectra at each concentration point.

Since concentrations are presented here in terms of the amount of PCR product rather than mole fraction of $\mathrm{C}$ and $\mathrm{T}$ alleles, it was necessary to subtract the component of $\mathrm{C}$ allele peak area that was contributed by the heterozygous sample (Figure 3 ). This peak area contribution was determined by multiplying the $\mathrm{T}$ allele peak area by the $\mathrm{C}: \mathrm{T}$ response factor determined from the heterozygote standard sample. This calculation was performed for peak areas determined from each individual

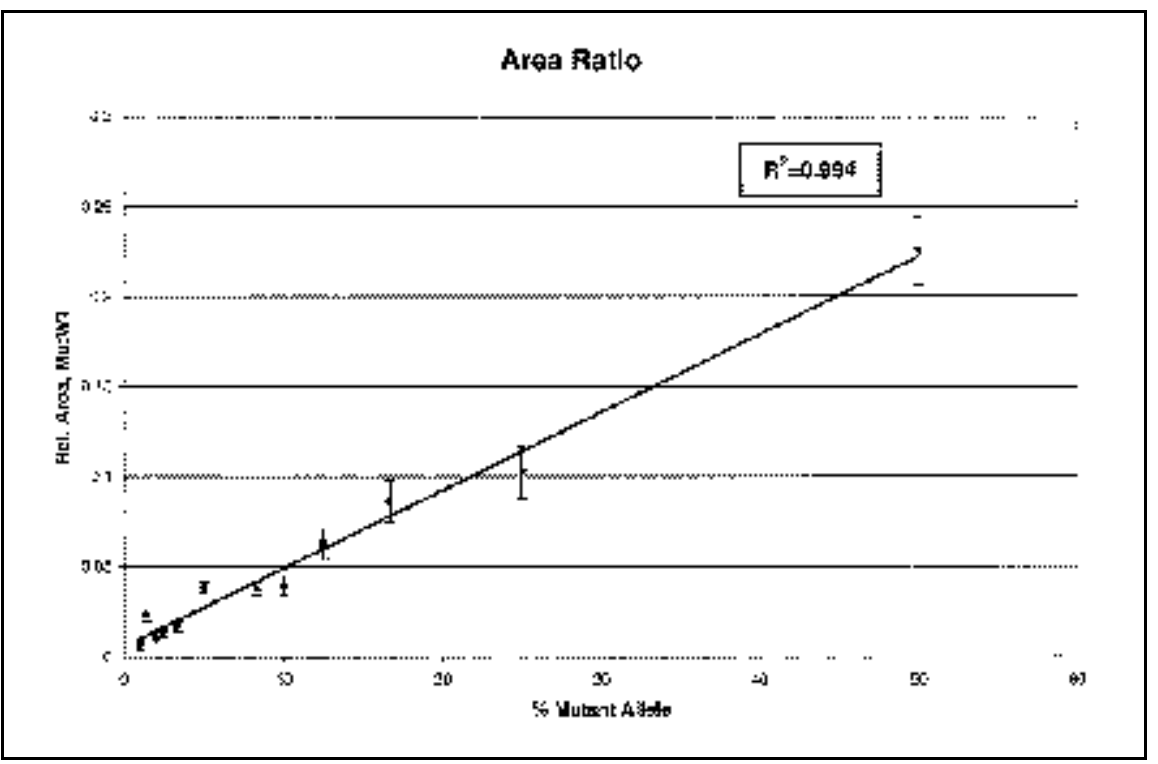

Figure 3. Quantitation curve for analysis of CP450 2D6 SNP site in pooled PCR products. Peak area ratios ( $\mathrm{T}$ allele: $\mathrm{C}$ allele) were measured from five replicate mass spectra and averaged, including spectra shown in Figure 2. Vertical bars show standard deviation at each concentration point. Area ratios were corrected for the relative response between $\mathrm{T}$ and $\mathrm{C}$ alleles as determined from analysis of replicate heterozygous mutant samples. 
spectrum, and then corrected peak areas were averaged for each concentration point. Corrected peak areas were then plotted and analyzed (Microsoft ${ }^{\circledR}$ Excel ${ }^{\circledR} 97$ ), yielding very good correlation between mass spectral response and relative amounts of mutant DNA target. The measured $\mathrm{S} / \mathrm{N}$ values for relevant peaks in all spectra were at least $5: 1$. For data points from $50 \%$ to $5 \%$ mutant, the standard deviation $(90 \%$ confidence) of the measured peak areas was typically $10 \%$. For mixtures comprising less than 5\% mutant, the standard deviation approached $35 \%$ of the measured peak area ratio. From these values, a reasonable LOQ of 5\% mutant DNA can be stated. It is implicit in this type of analysis that control assays are performed to ensure that no background extension occurs in the absence of target (data not shown).

A similar quantitation study was conducted for a common polymorphism in the ALDOB gene. In this case, the assays were performed using a reaction mixture containing all four dideoxynucleotides, with ddATP carrying a FAM label. For this system, the wild-type was a GG homozygote and the mutant was an AG heterozygote. The FAM label contributes an additional $410 \mathrm{Da}$ to the A allele, as shown in Figure 4. A concentration study was performed (Figures 4 and 5) for standard mixtures of PCR products from previously genotyped homozygote and heterozygote DNA samples. Again, the average of five replicate spectra was used to obtain area ratio measurements, and peak areas were corrected for the relative response of $A$ and $G$ alleles. The data in Figure 5 show that the use of labeled dideoxynucleotides can also be a very suitable approach to deriving relative quantitation information. The linearity of the data and the estimates of LOD and LOQ were similar to those found in Figure 3. At 2\% mutant, the measured $\mathrm{S} / \mathrm{N}$ for the minority allele peak was at least 4 in all spectra, and the standard deviation remained within $15 \%$ down to the $5 \%$ mutant level. From these values, we estimate an LOD of $2 \%$ and an LOQ of $5 \%$ mutant.

A main advantage of MALDI-TOF MS-based genotyping is its ability to multiplex many primer extension assays within a single sample $(13,20)$.
The approaches described here make use of the same basic primer extension chemistry used in multiplex assays and therefore may also support multiplex analysis of low-abundance alleles. Multiplex PCR and primer extension assays were performed for the CP450 polymorphism and a polymorphism in human LDLR region by amplifying homozygote and heterozygote samples. Multiplex PCR products from heterozygous mutant and homozygote samples were combined in a $5 \%$ mutant:wild-type ratio (a mutant allele abundance of $2.5 \%$ ), and the mixture was then genotyped (Figure 6). The data show unambiguous detection of the low-abundance alleles for both loci tested. A quantitation study was not performed for the multiplex experi- ments; however, the data are presented here to provide a basis for future investigation. Specifically, it is shown that there is no significant reduction in detection limit in analysis of multiple low-abundance alleles.

\section{DISCUSSION}

Two related approaches were used to mass-separate allelic products for accurate quantitative measurement. In the first, a specific mixture of deoxy- and dideoxynucleotides was used so that alleles differed in size by one or more bases. The use of a specific mixture of deoxy- and dideoxynucleotides in this fashion requires one to consider the sequence downstream of the genotyping

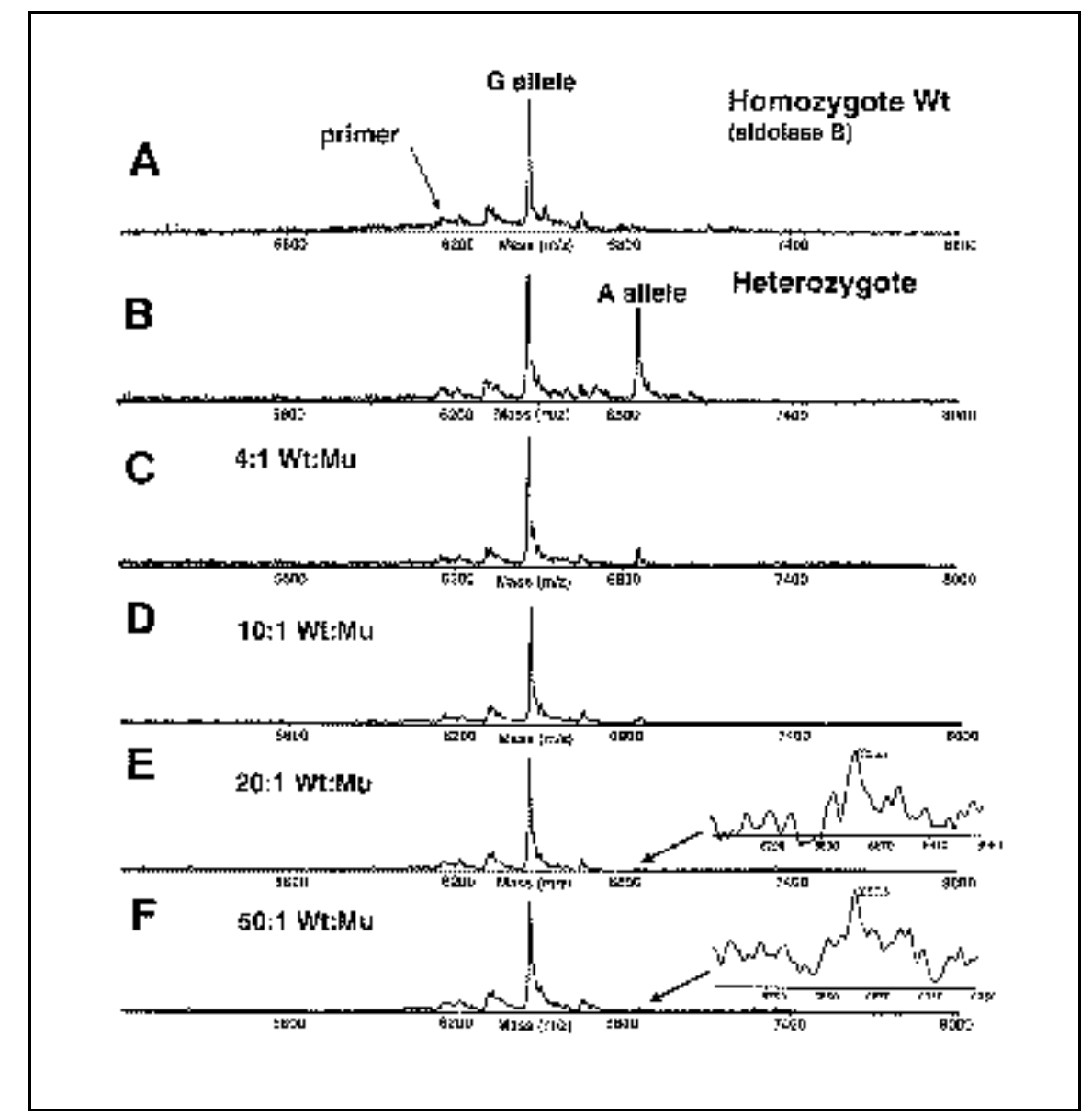

Figure 4. Mass spectra obtained at representative intervals from a quantitation study of ALDOB SNP site in pooled PCR products. Oligonucleotide sequences used were: PCR F: 5'-GAGCCCACG GCGTCTCTTC-3', PCR R: 5'-GAGACAGTGCCCAGGACAGAGTC-3', genotyping primer: 5'-TTTTATGACACCGTCATCAGCAG-3'. Mass spectra were generated from homozygous wild-type (A), heterozygous mutant (B) and mixtures of PCR from wild-type individuals and heterozygous mutant individuals in wild-type:mutant ratios ranging from 5:1-50:1 (C-F). In this example, the genotyping primer has been fully extended by ddG ( $\mathrm{G}$ allele) or FAM-ddA (A allele). 


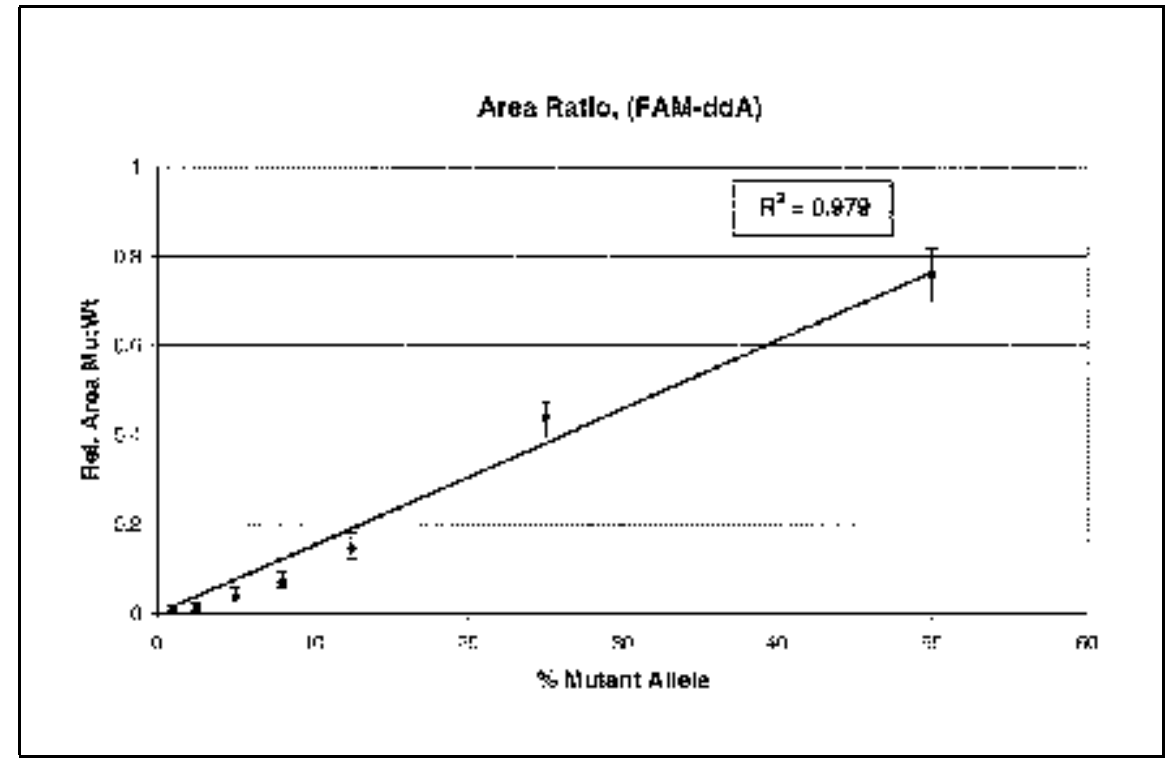

Figure 5. Quantitation curve for analysis of ALDOB SNP site in pooled PCR products, generated from data set including spectra shown in Figure 4. Peak area ratios (A allele:G allele) from five replicate mass spectra obtained across an entire sample spot were measured and averaged. Vertical bars show the standard deviation measured at each concentration point. Area ratios were corrected for the relative response between $\mathrm{A}$ and $\mathrm{G}$ alleles as determined from analysis of replicate heterozygous mutant samples. primer in designing the assay. Hence, the combination of deoxy- and dideoxynucleotides will often be quite different from one locus to the next. The second approach, by which the base corresponding to the allele under study is chosen to carry the FAM label, is more universal. Only the identity of the bases at the polymorphic site are required to assemble the correct dideoxynucleotide mixture to carry out the assay. Note that a variety of fluorescent and nonfluorescent labels spanning a mass range of 0-1000 Da can be used as mass separation labels, the main restriction being that some labels are undesirable because of production of multiple species during MALDI-TOF MS analysis. From the available suitably labeled dideoxynucleotides, one can assemble a universally applicable mixture of all four such that assay reaction products with well-resolved and readily quantitated allele peaks are generated.

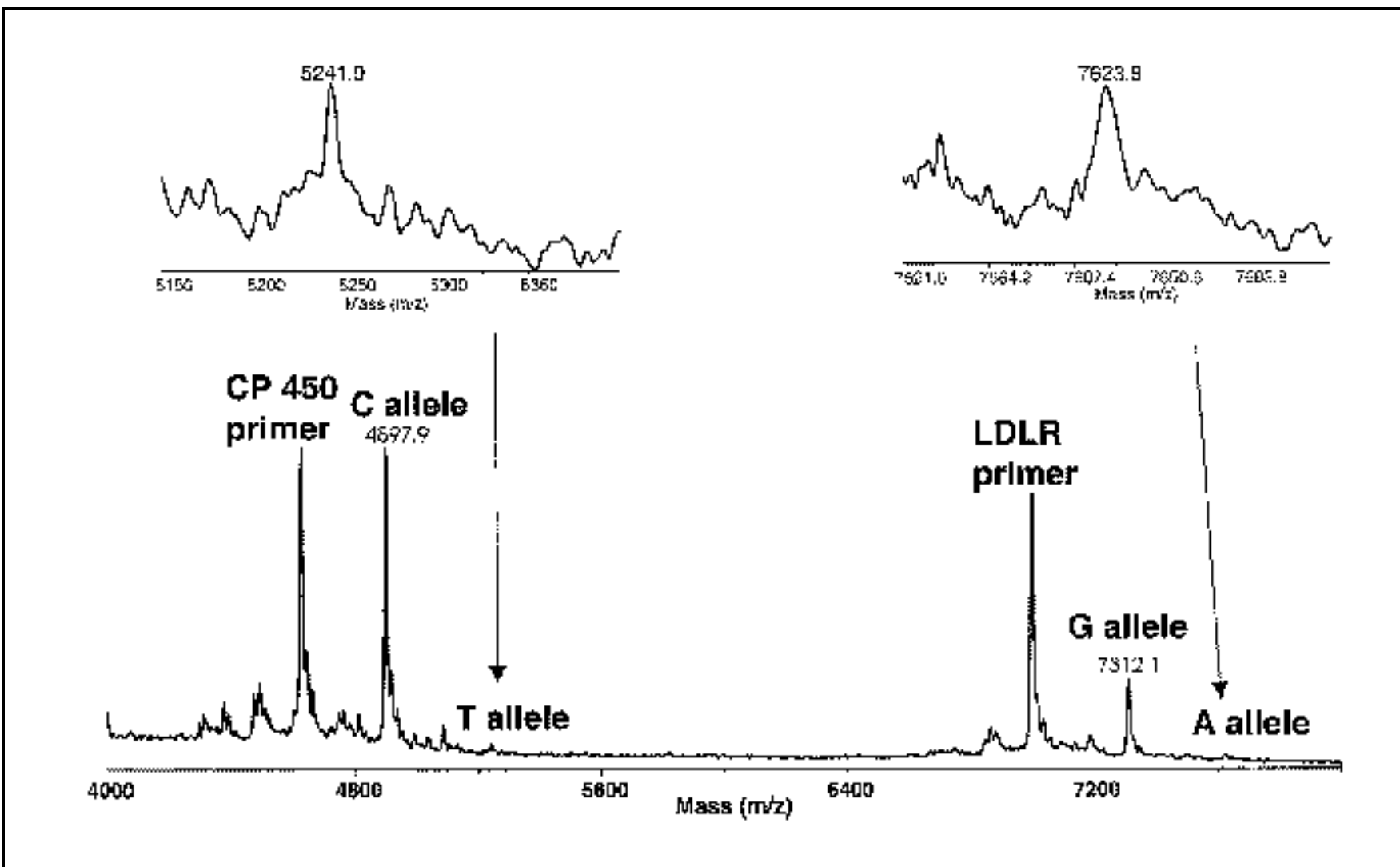

Figure 6. Illustration of multiplex genotyping of a DNA pool containing a mixture of twofold multiplex PCR products from CP450 2D6 and LDLR loci. Oligonucleotides used for LDLR genotyping were: PCR F: 5'-CAACACGGAGAAGCATTGTTTTC-3', PCR R: 5'-CTCAATCCTCATACTGACCTCTAC-3', genotyping primer: $5^{\prime}$-GGGCCAAGAAGGTATCTACC-3'. The PCR pool comprised individuals with $\mathrm{C}$ and $\mathrm{G}$ alleles at CP450 and LDLR loci, respectively, in a 20-fold excess over heterozygous wild-type:mutant and mutant:wild-type individuals at CP450 and LDLR, respectively. The genotyping reaction mixture contained ddC, ddG, dT and dA. 
The results presented here show a limit of detection of $2 \%$ mutant DNA and a limit of quantitation of 5\%. It is important to contrast these results with other techniques. The use of various fluorescence-based primer extension or minisequencing $(12,22,23)$ formats has been applied toward quantitation of allelic variants, primarily following RTPCR. For low-abundance genetic mutations in human genomic DNA amplified by PCR, a quantitative detection limit of between $0.4 \%$ and $1 \%$ can be observed. The use of PCR followed by the ligase detection reaction (PCR-LDR) detects $\mathrm{K}$-ras point mutations at a level of 1 in $500(0.2 \%)(16)$; however, it is not clear whether the range of quantitation of the PCR-LDR approach extends to such low levels. Most recently, the TaqMan assay has been described for minority allele detection in pooled samples (5). Accurate determination of allele frequency was observed for minority alleles at approximately $10 \%$ and $20 \%$; however, the ranges and limits of detection and quantitation were not explored for this approach. Despite somewhat lower sensitivity than the methods discussed here, the cost and throughput advantages of MALDI-TOF MS make this an ideal approach to validate putative SNP sites in a single experiment. It is evident that quantitative genotyping or allele detection in pools can be performed with ostensibly the same chemistry and MALDI-TOF instrumentation that is used for high-throughput analysis of SNPs from single individuals.

For the MALDI-based assay demonstrated here, the limit of detection of $2 \%$ is imposed primarily by random noise in the mass spectra. Accumulation and averaging of a greater number of individual MALDI spectra through the use of higher laser repetition rates may give a significant improvement in dynamic range without sacrificing acquisition time. Random noise also influences the limit of quantitation of this experiment. We observe that low-intensity peaks have a random noise component superimposed onto them. Thus, as peak area/ intensity decrease, the superimposed noise has an increasing effect on variability in peak shapes, height and area. Random noise also imparts minor deviations in the determination of the baseline in peak area measurements. These baseline deviations become significant when the $\mathrm{S} / \mathrm{N}$ drops to 5 or less. The use of increased averaging would therefore improve the quantitative range by keeping the degree of peak area deviation for low-abundance samples to within more acceptable levels.

Figure 6 shows that simultaneous analysis of multiple low-abundance alleles can be performed with a similar limit of detection (5\% shown in Figure 6). The extent of multiplexing when using termination mixtures comprising deoxyand dideoxynucleotides is limited by the sequence context at the regions of interest. Ideally, a combination producing extensions of +1 and +2 bases for wildtype and mutant alleles, respectively, should be used. Ultimately, as discussed here, using a universal mixture of four differently labeled, well-separated dideoxynucleotides would be more desirable. In either approach, a greater portion of the useful molecular weight range of the mass spectrometer is occupied by imparting the necessary separation between alleles. Thus, from consideration of the optimal mass range for oligonucleotide analysis, we expect that multiplex allele detection or quantitation up to 5 loci/sample is possible.

There are several applications in which the approach described may prove useful. First, the ability to measure an allele frequency in a single experiment can provide sufficient information in linkage disequilibrium analysis to determine the correlation of a given polymorphism to a particular disease state. Thus, a larger-scale sequencing or genotyping effort can be reduced to a small number of assays. Second, measurement of allele frequencies for putative SNPs following large scale sequencing efforts can establish whether the discovered variant is truly a high-frequency polymorphism or merely a sequencing error or a rare mutant. In this way, the efficacy of discovered SNPs as genetic markers can be tested in a single experiment. Finally, pools of clinical samples can be efficiently screened for common mutations (26). Groups of 10-50 DNA extracts can be screened for the presence of a given genotype, then only those pools show ing a positive result will be subdivided into smaller pools for further analysis such as sequencing. In the latter case, 
when the assay must only determine the presence of a mutant allele, quantitative information will not be required. A further reduction of labor and reagent consumption could then be achieved by pooling DNA samples before performing PCR.

\section{REFERENCES}

1.Armstrong, M., J.R. Idle and A.K. Daley. 1993. A polymorphic Cfo I site in exon 6 of the human cytochrome P450 CYPD6 gene detected by the polymerase chain reaction. Hum. Genet. 91:616-617.

2.Arnheim, N., C. Strange and H. Erlich. 1985. Use of pooled DNA samples to detect linkage disequilibrium of polymorphic restriction fragments and human disease: studies of the HLA class II loci. Proc. Natl. Acad. Sci. USA 82:6970-6974.

3.Barany, F. 1991. Genetic disease detection and DNA amplification using cloned thermostable ligase. Proc. Natl. Acad. Sci. USA 88:189-193.

4.Barcellos, L.F, W. Klitz, L.L. Field, R. Tobias, A.M. Bowcock, R. Wilson, M.P. Nelson, J. Nagatomi and G. Thomson. 1997. Association mapping of disease loci by use of a pooled DNA genomic screen. Am. J. Hum. Genet. 61:734-747.

5.Breen, G., D. Harold, S. Ralston, D. Shaw and D. St. Clair. 2000. Determining SNP allele frequencies in DNA pools. BioTechniques 28:464-470.

6.Brooks, C.C. and D.R. Tolan. 1993. Association of the widespread A149P hereditary fructose intolerance mutation with newly identified sequence polymorphisms in the aldolase B gene. Am. J. Hum. Genet. 52:835840.

7.Bruenner, B.A., T.T. Yip and T.W. Hutchens. 1996. Quantitative analysis of oligonucleotides by matrix-assisted laser desorption/ionization mass spectrometry. Rapid Commun. Mass Spectrom. 10:1797-1801.

8.Carmi, R., T. Rokhlina, A.E. Kwitek-Black, K. Eibedour, D. Nishimura, E.M. Stone and V.C. Sheffield. 1995. Use of a DNA pooling strategy to identify a human obesity syndrome locus on chromosome 15. Hum. Mol. Genet. 3:1331-1335.

9.Collins, F.S., A. Patrinos, E. Jordan, A. Chakravarti, R. Gesteland and L. Walters. 1998. New goals for the U.S. Human Genome Project: 1998-2003. Science 282:682-689.

10.Day, J.P., R.P. Hammer, D. Bergstrom and F. Barany. 1999. Nucleotide analogs and new buffers improve a generalized method to enrich for low abundance mutations. Nucleic Acids Res. 27:1819-1827.

11.Fei, Z., T. Ono and L.M. Smith. 1998. MALDI-TOF mass spectrometric typing of single nucleotide polymorphisms with masstagged ddNTPs. Nucleic Acids Res. 26:28272828.

12.Greenwood, A.D. and D.T. Burke. 1996. Single nucleotide primer extension: quantitative range, variability, and multiplex analysis.
Genome Res. 6:336-348.

13.Haff, L.A. and I.P. Smirnov. 1997. Single nucleotide polymorphism identification assays using a thermostable DNA polymerase and MALDI-TOF MS. Genome Res. 7:378388.

14.Haff, L.A. and I.P. Smirnov. 1997. Multiplex genotyping of PCR products using mass tag-labeled primers. Nucleic Acids Res. 25:3749-3750.

15.Juhasz, P., M.T. Roskey, I.P. Smirnov, L.A. Haff, M.L. Vestal and S.A. Martin. 1996. Applications of delayed extraction matrix-assisted laser desorption/ionization time-offlight mass spectrometry to oligonucleotide analysis. Anal. Chem. 68:941-946.

16.Khanna, M., P. Park, M. Zirvi, W. Cao, A. Picon, J. Day, P. Paty and F. Barany. 1999. Multiplex PCR/LDR for detection of K-ras mutations in primary colon tumors. Oncogene 18:27-38.

17.Kruglyak, L. 1999 Prospects for wholegenome linkage disequilibrium mapping of common disease genes. Nat. Genet. 17:21-24.

18.Landegren, U., M. Nilsson and P.-Y. Kwok. 1998. Reading bits of genetic information: methods for single-nucleotide polymorphism analysis. Genome Res. 8:769-776.

19.Little, D.P., A. Braun and H. Koster. 1997. Detecting CFTR gene mutations by using primer oligo base extension and mass spectrometry. Clin. Chem. 43:1151-1158.

20.Risch, N. and J. Teng. 1998. The relative power of family-based and case-control designs for linkage disequilibrium studies of complex human diseases I. DNA pooling. Genome Res. 8:1273-1288.

21.Ross, P., L. Hall, I.P. Smirnov and L. Haff. 1998. High level multiplex genotyping by MALDI-TOF mass spectrometry. Nat. Biotechnol. 16:1347-1351.

22.Syvänen, A.-C. 1999. From gels to chips: "minisequencing" primer extension for analysis of point mutations and single nucleotide polymorphisms. Hum. Mutat. 13:1-10.

23.Syvänen, A.-C., E. Ikonen, T. Manninen, M. Bengtstrom, H. Soderlund, P. Aula and L. Peltonen. 1994. Convenient and quantitative detection of the frequency of a mutant allele using solid-phase minisequencing: application to aspartylglucosaminuria in Finland. Genomics 12:590-595.

24.Wang, D.G., J.-B Fan, C.-J. Siao, A. Berno, P. Young, R. Sapolsky, G. Ghandour, N. Perkins, E. Winchester, J. Spencer et al. 1998. Large scale identification, mapping, and genotyping of single nucleotide polymorphisms in the human genome. Science 280:1077-1082

25.Warnich, L., M.J. Kotze, E. Langenhoven and A.E. Retief. 1992. Detection of a frequent polymorphism in exon 10 of the lowdensity lipoprotein receptor gene. Hum. Genet. 89:362.

26.Zarbl, H., C. Aragaki and L.P. Zhao. 1998. An efficient protocol for rare mutation genotyping in a large population. Genetic Testing 2:315-321.

27.Zhao, L.P., C. Aragaki, L. Hsu and F. Quiaoit. 1998. Mapping of complex traits by single-nucleotide polymorphisms. Am. J. Hum. Genet. 63:225-240.
Received 27 September 1999; accepted 10 May 2000.

Address correspondence to:

Dr. Philip Ross

Applied Biosystems

500 Old Connecticut Path

Framingham, MA 01701, USA

e-mail:philip_ross@pbio.com 